\title{
Simulation of interaction between a freely moving solid particle and a freely moving liquid droplet by lattice Boltzmann method
}

Bo Yang ${ }^{1}$, Sheng Chen ${ }^{* 2,3}$

1 State Key Laboratory of Coal Combustion, School of Energy and Power aEngineering, Huazhong University of Science and Technology, Wuhan, 430074, China

2 Faculty of Engineering, University of Nottingham, University Park, Nottingham NG7 2RD, UK

3 Institute for Modelling and Simulation in Fluodynamics, Nanoscience and Industrial Mathematics "Gregorio Millan Barbany", Universidad Carlos III de Madrid, Leganes 28911, Spain

* Corresponding author at: Faculty of Engineering, University of Nottingham, UK. Email: sheng.chen@ nottingham.ac.uk

\begin{abstract}
Solid particles and liquid droplets widely coexist in many industrial processes. Particle-droplet interactions critically influence the dynamics of those processes. In this study, the interactions between a freely moving solid particle and a freely moving liquid droplet are numerically investigated using the lattice Boltzmann method. Until now the open literature on such topic is quite sparse. Through the present numerical investigation, two regimes to classify the interactions between a freely moving solid particle and a freely moving liquid droplet are proposed. In addition, it is found that the particle-to-droplet size ratio and particle's wettability play critical roles in such interactions.
\end{abstract}

Key words: Particle-droplet interaction; collision; agglomeration; separation; lattice Boltzmann method.

\section{Introduction}

Liquid-gas-particle (LGP) systems widely exist in many industrial applications such as electronics fabrication by ink-jet printing [1] and emulsion stabilization in food [2], cosmetics [3], and petroleum industries [4,5]. In LGP systems, the interactions between droplets and particles critically influence the performance of the systems. Up to date, the particle-particle interactions have been investigated extensively [6-10]. A so-called DKT (drafting, kissing, and tumbling) process may happen during interaction between two solid particles. For collisions between two droplets, many studies [11-17] observed four types of collision regimes: bouncing, coalescence, stretching and reflexive, based on the Weber number (We) and impact parameter (b). Compared with its particle-particle and droplet-droplet counterparts, the open studies on particle-droplet interactions are relatively sparse. Shen et al. [18] analyzed the effects of the droplet's velocity, size ratio of the droplet to solid, and temperature difference on liquid attachment during droplet-particle collisions. The authors found that the percentage of liquid attachment decreases against increasing droplet impact velocity. In addition, the authors concluded that a center-to-center collision would result in more liquid attachment than an off-center collision. Mitra et al. [19] investigated the collision behavior of a small solid particle against a large stationary droplet. They analyzed the processes of the solid particle penetrating 
through the droplet and claimed that the capillary force dominated the whole process. Gac et al. [20, 21] investigated the influences of some dimensionless parameters, such as the Weber number, capillary number and droplet-to-particle diameter ratio, on the kinetics of central collision between a droplet and a particle. The authors observed three types of collision behavior: coalescence, ripping and coating, and skirt scattering. The authors also found that the behavior of central collision were nearly insensitive to the particle's shape. Hardalupas et al. [22] investigated the dynamics of small droplets impacting against a large solid surface. Their results showed that an impinging droplet could form a crown, which was influenced by surface roughness, droplet kinematics and liquid properties. Bakshi et al. [23] conducted experiments to investigate the effects of droplet Reynolds number and particle-to-droplet size ratio on the behavior of a liquid film on a solid surface. Three transitional phases of the film kinetics, namely the initial drop deformation phase, inertia dominated phase, and viscosity dominated phase, were observed. Fakhari et al.[24] conducted a simulation for a droplet's impingement on a hydrophilic or superhydrophobic cylinder under gravity. Their results indicated that a droplet tended to adhere to the surface of a hydrophilic cylinder while it tended to break up and detach from the surface of a hydrophobic cylinder. Malgarinos et al. [25] simulated the impact processes of a liquid droplet against a spherical stationary solid particle. Two different regimes: the partial/full rebound and coating regime were identified. Malgarinos et al. [26]-[27] investigated the collisions between heavy gasoil droplets and solid catalytic particles. In their studies, phase-change phenomena and catalytic cracking surface reactions were taken into account.

In the all above-mentioned studies, the investigated solid particles or liquid droplets are stationary, rather than freely moving. For the scenarios where both a particle and a droplet can freely move, Dubrovsky et al. [25] carried out a pioneering experimental study on particle-droplet collisions. For the cases where the droplet-to-particle size ratio was greater than 1, they observed four collision modes: particle capture, "shooting through" with satellite droplet formation, "shooting through" with gas bubble formation and droplet destruction. Then, Deen et al. [30] simulated collisions of a falling particle against a rising bubble by a front tracking approach combined with an immersed boundary (IB) scheme. They studied the effect of particle's density on the interaction between the particle and the bubble. Higher particle's density led to more significant deformation of the bubble and increasing the possibility of the particle penetrating through the bubble. In their work, only head-on collisions were considered. Sasic et al. [31] numerically studied the interaction between settling particles and rising microbubbles. They observed that the particles would attach to the bubbles when the initial horizontal distance between their centers was small enough, while the particles would pass through the bubbles if such initial distance was large. Kan et al. [32] simulated the processes of particle-particle combination by a droplet. The authors discussed the effect of particle's wettability on the critical velocity for particle-particle combination. They concluded that such critical velocity varied non-monotonically with the particle's wettability. They also investigated the effect of droplet size on the adhesiveness of two colliding particles [33]. It was found that against the increasing of droplet's diameter, the adhesiveness of particles became weak. Recently, Pawar et al. [34] experimentally investigated particle-droplet collisions at low capillary numbers. The collision behavior was classified into two regimes: agglomeration (merging) and stretching separation (breaking), based on the Weber number and impact parameter. In addition, they proposed a map based on the droplet-to-particle size ratio to illustrate the regimes. 
Through the above literature survey, it is clear that the open literature on interactions between a freely moving droplet and a freely moving particle is extremely sparse. In addition, the in-depth knowledge about the effect of particle's wetting property on particle-droplet collisions is still absent. To bridge the gap, in this study, the interactions between a freely moving particle and a freely moving droplet are investigated numerically. Moreover, the effects of particle's wettability on collision behavior are also discussed.

\section{Numerical method}

It is difficult to conduct experiments to study interactions between a freely moving solid particle and a freely moving droplet, so numerical methods are chosen for the present research. Until now the lattice Boltzmann method (LBM) has matured to model particle dynamics [6-10] and multi-phase multi-component flow [35-[40]. Consequently, in this work the LBM-based Shan-Chen multi-phase multi-component (MCMP) model [35] is adopted for simulating liquid droplets and the LBM-based particle dynamics modelling approach [41] is employed for simulating particle's motion.

\subsection{Multiphase flow}

In the MCMP model, the pseudo fluid particle distribution function (PDF) for each fluid component satisfies [35]:

$$
f_{i}^{\sigma}\left(\mathbf{x}+\mathbf{e}_{i} \Delta t, t+\Delta t\right)=f_{i}^{\sigma}(\mathbf{x}, t)-\frac{1}{\tau_{\sigma}}\left(f_{i}^{\sigma}(\mathbf{x}, t)-f_{i}^{\sigma, e q}(\mathbf{x}, t)\right)
$$

where $f_{i}^{\sigma}(\mathbf{x}, t)$ is the PDF for the $\sigma$ th fluid component and $\tau_{\sigma}$ is its relaxation time, which is determined by the kinematic viscosity as $v_{\sigma}=c_{s}^{2}\left(\tau_{\sigma}-0.5 \Delta t\right) . f_{i}^{\sigma, e q}(\mathbf{x}, t)$ is the equilibrium distribution function and can be expressed as

$$
f_{i}^{\sigma, e q}(\mathbf{x}, t)=\omega_{i} \rho_{\sigma}\left[1+\frac{\mathbf{e}_{i} \cdot \mathbf{u}_{\sigma}^{e q}}{c_{s}^{2}}+\frac{\left(\mathbf{e}_{i} \cdot \mathbf{u}_{\sigma}^{e q}\right)^{2}}{2 c_{s}^{4}}-\frac{\mathbf{u}_{\sigma}^{e q 2}}{2 c_{s}^{2}}\right]
$$

where $\omega_{0}=4 / 9, \omega_{1-4}=1 / 9$ and $\omega_{5-8}=1 / 36$, and $\mathbf{e}_{i}$ is the discrete velocity. For the D2Q9 model $\mathbf{e}_{i}$ is defined as

$$
\mathbf{e}_{i}=\left\{\begin{array}{cc}
(0,0) & i=0 \\
c(\cos [(i-1) \pi / 2], \sin [(i-1) \pi / 2]) & i=1,2,3,4 \\
\sqrt{2} c(\cos [(2 i-1) \pi / 4], \sin [(2 i-1) \pi / 4]) & i=5,6,7,8
\end{array}\right.
$$

In this paper $c=\Delta x=\Delta t=1$. The macroscopic density $\rho_{\sigma}$ and velocity $\mathbf{u}_{\sigma}$ for the $\sigma$ th fluid component are obtained by

$$
\rho_{\sigma}=\sum_{i} f_{i}^{\sigma}, \mathbf{u}_{\sigma}=\frac{1}{\rho_{\sigma}} \sum_{i} \mathbf{e}_{i} f_{i}^{\sigma}
$$

The equilibrium velocity $\mathbf{u}_{\sigma}^{e q}$ is calculated by

$$
\mathbf{u}_{\sigma}^{e q}=\mathbf{u}^{c}+\frac{\tau_{\sigma} \mathbf{F}_{\sigma}}{\rho_{\sigma}}
$$

where $\mathbf{u}^{\mathrm{c}}$ is the composite macroscopic velocity and given by 


$$
\mathbf{u}^{c}=\frac{\sum_{\sigma} \frac{1}{\tau_{\sigma}} \sum_{i} f_{i}^{\sigma} \mathbf{e}_{i}}{\sum_{\sigma} \frac{\rho_{\sigma}}{\tau_{\sigma}}}
$$

The pressure $P$ is obtained by

$$
P=\left(\rho_{\sigma}+\rho_{\bar{\sigma}}\right) c_{s}^{2}+3 G c^{2} \rho_{\sigma} \rho_{\bar{\sigma}}
$$

The inter-particle force on the $\sigma$ th component is defined as [37]

$$
F_{\mathrm{int}, \sigma}(\mathbf{x}, \mathrm{t})=-G \rho_{\sigma}(\mathbf{x}, \mathrm{t}) \sum_{i} \omega_{i} \rho_{\sigma}\left(\mathbf{x}+\mathbf{e}_{i} \Delta \mathrm{t}, \mathrm{t}\right) \mathbf{e}_{i}
$$

where the $\sigma$ and $\bar{\sigma}$ indicate two different fluid components and $G$ is a parameter that controls the interaction strength of inter-particle force.

Solid surface forces are incorporated into the MCMP model through

$$
F_{\text {ads }, \sigma}(\mathbf{x}, \mathrm{t})=-G_{\text {ads }, \sigma} \rho_{\sigma}(\mathbf{x}, \mathrm{t}) \sum_{i} \omega_{i} s\left(\mathbf{x}+\mathbf{e}_{i} \Delta \mathrm{t}, \mathrm{t}\right) \mathbf{e}_{i}
$$

where $s$ is an indicator function, which equals to 1 or 0 for a solid or fluid node, respectively. The parameter $G_{a d s}$ controls the interaction strength between fluid and solid nodes.

To reduce the spurious velocity currents, we adopt the improvement scheme of sufficient isotropy (up to 8 order) to calculate the gradient of the density [36] and the explicit forcing (EF) scheme to discrete the inter-particle force $[38,40]$.

\subsection{Particle treatment}

In this work we adopt the half-way bounce back scheme for particle boundary treatment as it can guarantee mass conservation during simulation. Boundary nodes are located at the midway between a fluid node and a solid node. The normal streaming step happens when pseudo fluid particles stream from a fluid node to a neighboring fluid node. If the adjacent node of a fluid node is solid, the half-way bounce-back scheme acts as

$$
f_{\bar{i}}(\mathbf{x}, t+1)=f_{i}^{+}-6 \rho_{w} \omega_{i}\left(\mathbf{u}_{b} \cdot \mathbf{e}_{i}\right)
$$

where $\bar{i}$ indicates the direction opposite to $i, \mathbf{u}_{b}$ is the velocity of the boundary node and $\rho_{w}$ is set to be the density of external fluid node. The second term at the right hand of Eq. (10) represents the change of PDF due to wall-fluid interaction. The force and torque exerting on a particle are calculated by

$$
\begin{aligned}
& F_{b}=\sum_{b n} \sum_{\sigma} \mathbf{e}_{\bar{i}}\left(2 f_{\bar{i}, \sigma}^{+}\left(x+\mathbf{e}_{i} \Delta t, t\right)-6 \rho_{\sigma}\left(x+\mathbf{e}_{i} \Delta t, t\right) \omega_{i}\left(\mathbf{u}_{b} \cdot \mathbf{e}_{\bar{i}}\right)\right) \\
& T_{b}=\sum_{b n}\left(\mathbf{r}_{b n}-\mathbf{R}\right) \sum_{\sigma} \mathbf{e}_{\bar{i}}\left(2 f_{\bar{i}, \sigma}{ }^{+}\left(\mathbf{x}+\mathbf{e}_{i} \Delta t, t\right)-6 \rho_{\sigma}\left(\mathbf{x}+\mathbf{e}_{i} \Delta t, t\right) \omega_{i}\left(\mathbf{u}_{b} \cdot \mathbf{e}_{\bar{i}}\right)\right)
\end{aligned}
$$

where $\mathbf{r}_{\mathrm{bn}}$ and $\mathbf{R}$ are the distance between a boundary node and the center of the particle, and the radius of the particle, respectively. The force and torque exerting on a particle due to interaction between fluid and solid surface are obtained by

$$
\begin{aligned}
\mathbf{F}_{a d} & =\sum_{b n} \sum_{\sigma} G_{a d s, \sigma} \omega_{i} \rho_{\sigma}\left(\mathbf{x}+\mathbf{e}_{i} \Delta t, t\right) \mathbf{e}_{\bar{i}} \\
T_{a d} & =\sum_{b n}\left(\mathbf{r}_{b n}-\mathbf{R}\right) \sum_{\sigma} G_{a d s, \sigma} \omega_{i} \rho_{k}\left(\mathbf{x}+\mathbf{e}_{i} \Delta t, t\right) \mathbf{e}_{\bar{i}}
\end{aligned}
$$

The fluid domain will be updated after the particle moves: some fluid nodes will be covered by the particle and some solid nodes will change to fluid nodes, which will lead to momentum exchange between the particle and fluid. The impulse force and torque exerting on the particle due to particle movement are computed by [41] 


$$
\begin{aligned}
\mathbf{F}_{i m} & =\sum_{C} \sum_{\sigma} \rho_{\sigma} \mathbf{u}_{c}-\sum_{U} \sum_{\sigma} \rho_{b} \mathbf{u}_{b} \\
T_{i m} & =\sum_{C} \sum_{\sigma}\left(\mathbf{r}_{C}-\mathbf{R}\right) \rho_{\sigma} \mathbf{u}_{c}-\sum_{U} \sum_{\sigma}\left(\mathbf{r}_{U}-\mathbf{R}\right) \rho_{b} \mathbf{u}_{b}
\end{aligned}
$$

where $C$ and $U$ denote the covered and new fluid nodes, respectively, and $\rho_{b}$ is the average density of neighboring fluid nodes.

When a solid particle moves across lattice nodes, some solid nodes will become to new fluid nodes. As in this algorithm the interior fluid is excluded, fluid information is unknown at the new fluid nodes, and therefore a refill procedure is required. We use the direct extrapolation of PDF for new fluid nodes.

The total force and torque exerting on a particle, $\mathbf{F}_{\mathrm{p}}, T_{\mathrm{p}}$, are the sum of $\mathbf{F}_{\mathrm{b}}, \mathbf{F}_{\mathrm{ad}}, \mathbf{F}_{\mathrm{im}}$ and $T_{\mathrm{b}}, T_{\mathrm{ad}}, T_{\mathrm{im}}$, respectively. According to the Newtonian dynamic law, the following equations are solved to capture the motion of a particle.

$$
\begin{aligned}
& M_{p} \frac{d \mathbf{U}_{p}(t)}{d t}=\mathbf{F}_{p}(t) \\
& I_{p} \cdot \frac{\mathbf{\Omega}_{p}(t)}{d t}+\mathbf{\Omega}_{p}(t) \times\left[\mathbf{I}_{p} \cdot \mathbf{\Omega}_{p}(t)\right]=T_{p}(t)
\end{aligned}
$$

where $M_{p}, I_{p}$ are the mass and inertial tensor of the particle, $\mathbf{F}_{p}(\mathrm{t})$ and $T_{p}(\mathrm{t})$ are the total force and toque exerting on the particle, and $\mathbf{U}_{p}(t)$ and $\boldsymbol{\Omega}_{p}(t)$ are the translational and angular velocity of the particle, respectively.

\section{Model validation}

\subsection{Contact line motion on a stationary circular cylinder}

The contact line motion on a single circular cylinder has been simulated by Shao et al. [42] and $\mathrm{Li}$ et al. [43]. It is used here to validate the capability of our computer code for simulating complicated wetting phenomena. A stationary cylinder with a dimensionless radius of 40 is fixed at the center of a fluid domain and the size of the domain is $200 \times 200$. Periodic boundary conditions are applied at the left and right boundaries and neutral wetting conditions are adopted for the upper and lower walls. Initially, the lower half part of the domain is occupied by fluid 1 with density of 2.05 while fluid 2 , whose density reads 0.01 , occupies the rest space. The kinematic viscosity of fluid 1 and fluid 2 both are 0.167 . We set the fluid-fluid interaction strength $G_{12}$ to be 0.2 and adjust the fluid-solid interaction strength $G_{a d s}$ to achieve various wetting conditions. Three choices: $G_{a d s, 1}=-G_{a d s, 2}=-0.05, G_{a d s, 1}=-G_{a d s, 2}=0.0$ and $G_{a d s, 1}=-G_{a d s, 2}=0.05$ are used and they lead the contact angle to be 60, 90 and 120 degree, respectively. Fig. 1 shows the contact angles of the stationary cylinder under these three wetting conditions, which agree well with the previous predicted results [43].
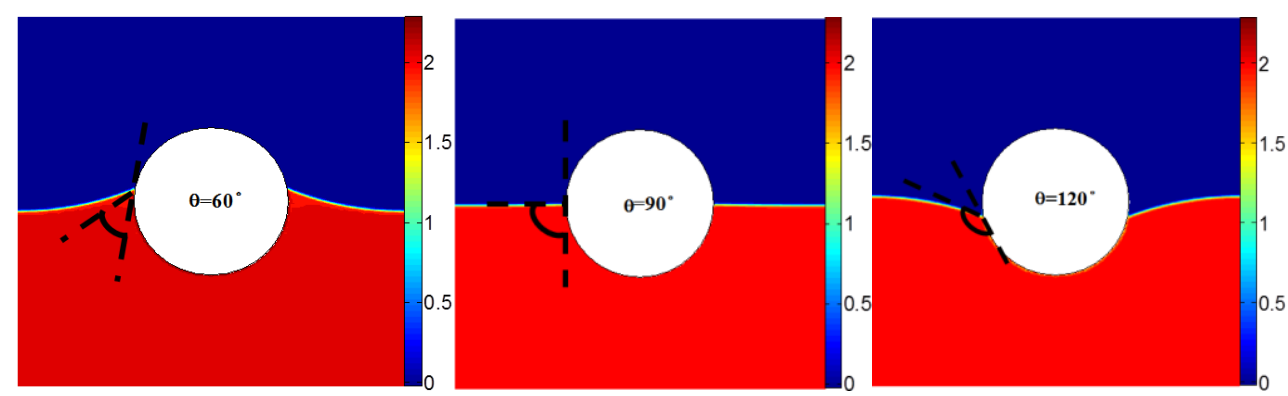
Fig. 1 The contact angles on a stationary cylinder under three different wetting conditions.

Red: fluid 1; Blue: fluid 2; White: solid cylinder. The color bars indicate the densities of fluids.

\subsection{Dynamics of single solid particle at liquid-vapor interface}

We simulate the motion of a single solid particle at the liquid-vapor interface to validate the capability of our computer code for modelling the interaction between moving particles and fluids. The grid resolution for the investigated domain is $101 \times 101$, and the particle, whose dimensionless radius reads 6 , is initially located on the center of the domain. The fluid-fluid interaction strength $G_{11}$ is set to be -0.65 and the fluid-solid interaction strength $G_{s}$ is -0.04 . The kinematic viscosities of the fluids both are 0.167 . At the beginning, the lower half of the domain is occupied by fluid 2 whose density is 2.54 and the upper half of the domain is occupied by fluid 1 whose density is 0.01 . The solid particle's density reads 5.0. The parameters and conditions used in this simulation are the same as those adopted in [41]. As shown in Fig. 2, we observe that the equilibrium contact angle $\theta$ reads 77.46, which agrees well with that reported in [41]. In the equilibrium state, the liquid-liquid interface is flat, which is consistent with the conclusion of Onishi et al. [44].

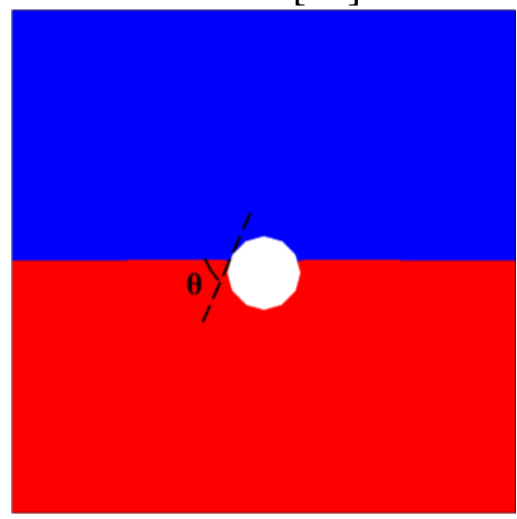

Fig. 2 Equilibrium contact angle at 50000 steps.

\section{Results and discussion}

In this section, we investigate the interaction between a freely moving solid solid particle and a freely moving liquid droplet. The investigated domain is illustrated by Fig. 3. In order to reduce the research complicity, here only a two-dimensional domain is investigated. The initial radius of the liquid droplet (marked in red) $R_{\mathrm{d}}=20$. The droplet is made up by fluid 2 , whose density reads 2.33 . The radius of the solid particle (depicted in white) is $R p=20$ (namely $R p / R d=1.0$ ) but its density is 5.0. The rest space of the domain is filled with fluid 1 whose density reads 2.33 . The particle and the droplet move oppositely and their initial velocities are $U_{0}$ and $-U_{0}\left(U_{0}=0.1\right)$, respectively. After $\mathrm{t}-=0$, the particle and the droplet keep on moving due to their inertia. The size of simulation domain is $401 \times 201$. Periodic boundary conditions are adopted for the upper and lower boundaries. The initial horizontal distance between the particle and the droplet is $L=3 R_{\mathrm{d}}=60$. The eccentricity ratio $B=\Delta y /\left(R_{p}+R_{d}\right)$, where $\Delta y$ is the initial vertical distance between the droplet and the particle. The interaction strength between two fluid components is $G_{12}=0.2$ and the corresponding surface tension is $\sigma=0.112$. The Weber number of the droplet is $W e=4 \rho_{\mathrm{d}} D_{\mathrm{d}} U_{0}^{2} / \sigma=33.3$, where $D_{\mathrm{d}}$ is the diameter of the droplet. The above parameters all are in lattice units. 


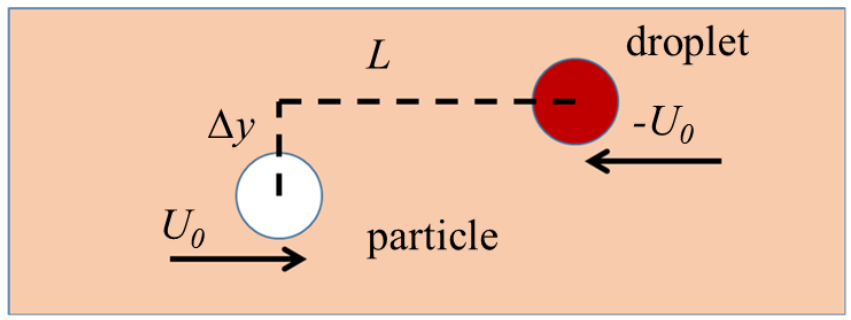

Fig. 3 Schematic diagram of the investigated domain.

Based on the numerical results of particle-droplet interaction at various $B$, an agglomeration regime and a separation regime are proposed in this work. Meanwhile the effect of the particle's wettability is considered. It is found that the particle's wettability can lead to obviously different phenomena, which will be discussed in detail below.

\subsection{Agglomeration regime}

When $B=0$, namely central collision, a particle and a droplet will experience an agglomeration process, in which the particle and the droplet will merge together. Fig. 4 shows the collision process between a neutral particle and a droplet at $B=0$. In this paper, the lattice time is normalized by $2 R_{d} / U_{0}$. It can be observed that the droplet firstly stretches in the vertical direction, and its left side becomes nearly flat until it attaches to the particle. Then the droplet becomes long and thin in the horizontal direction, indicating that the capillary force overcomes the droplet surface tension. Later, the droplet recovers its circular shape slowly, and moves along with the neutral particle. Finally the neutral particle and the droplet form a stable combination and a contact angle of 90 degree is observed between their interfaces.

The variation of the neutral particle's horizontal velocity is shown in Fig. 5. The particle's velocity is normalized by $U_{0}$. As shown in Fig. 5 (b), the horizontal velocity decreases linearly when $t<0.2$ due to the hydrodynamic resistance force. Then it increases linearly when $0.2<t<0.4$, which results from an attraction effect due to capillary force. As the droplet stretches in the vertical direction, the horizontal velocity experiences a decreasing process at $0.4<t<2.0$. During the process of the droplet attaching to the neutral particle $(2.0<t<5.0)$, the horizontal velocity increases slowly with some fluctuations. As shown in Fig. 5 (a), since $t>5.0$ the horizontal velocity decreases slowly with fluctuations. Fig. 5 (c) depicts the variation of drag force of the neutral particle. The drag force experiences significant oscillations. To illustrate the variation trend of the drag force more clearly, the drag force curve is smoothed by the polynomial interpolation method, as shown in Fig. 5 (d). For $0<t<0.2$ the resistance $(F x<0)$ dominates the motion of the neutral particle, which causes the reduction of the horizontal velocity, as shown in Fig. 5 (b). Hower, during $0.2<t<0.4$ the accelerating force $(F x>0)$ dominates the movement of the neutral particle. This accelerating force may stem from capillary force, which has an attractive effect due to the presence of the fluid-fluid interface [45]. During $0.4<t<5.0$, the drag force fluctuates obviously around 0 , which corresponding to the process of the droplet approaching to the neutral particle. When $t>5.0$, the drag force approaches to 0 slowly with very weak fluctuations. 


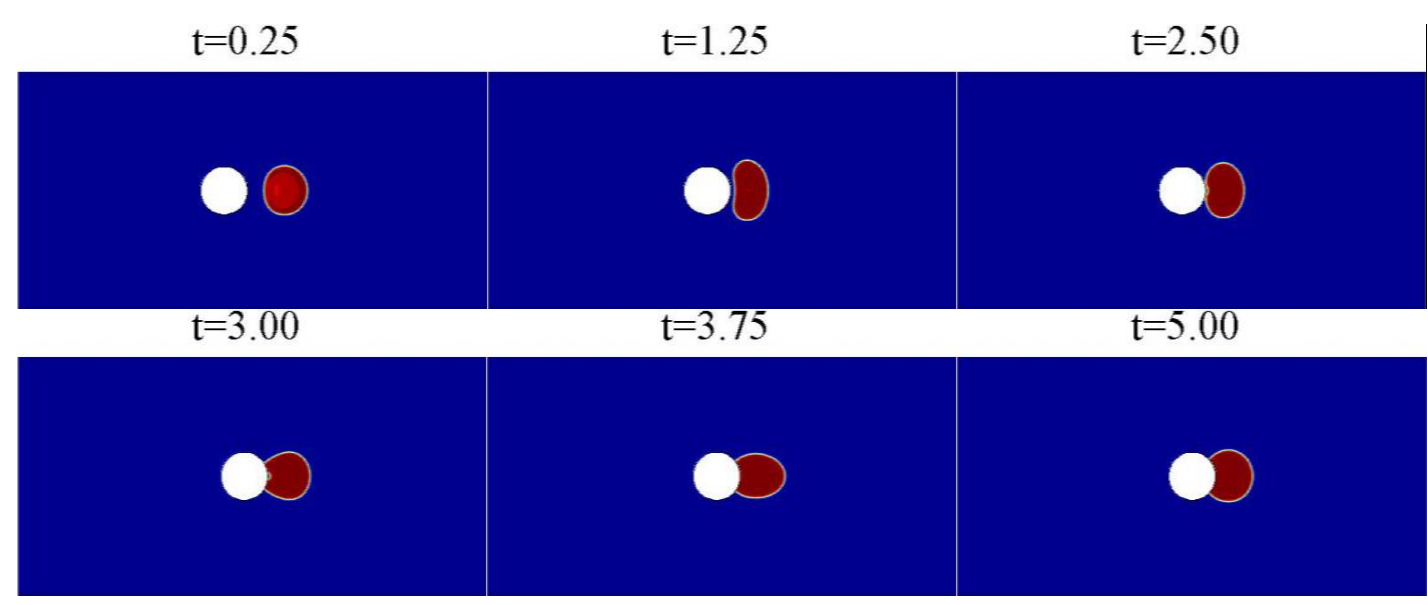

Fig. 4 The collision process between a neutral particle and a liquid droplet at $B=0$, in which the white cylinder represents the particle and the red deformable represents the droplet.
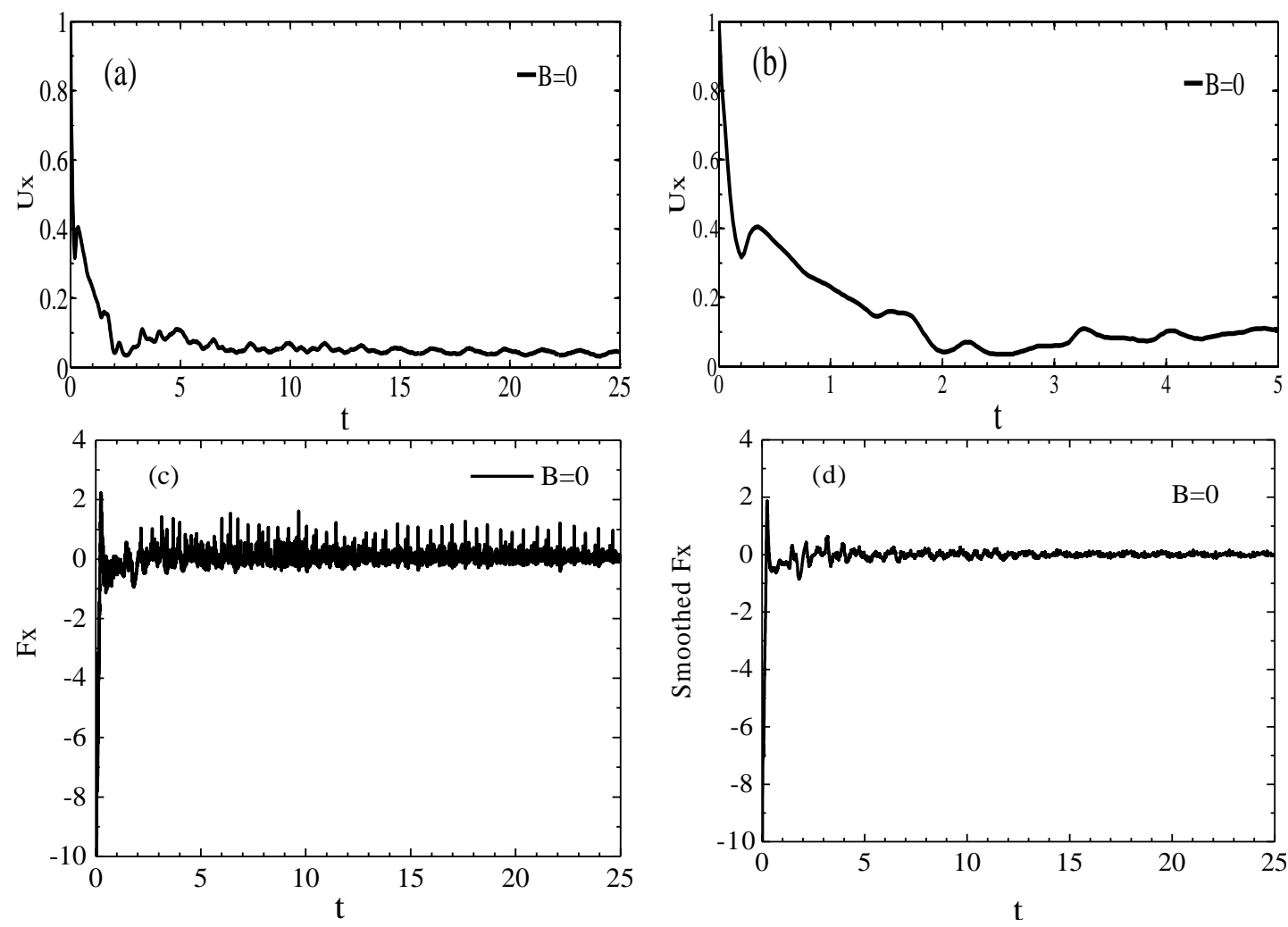

Fig. 5 (a) Variations of horizontal velocity of the neutral particle; (b) Enlarged figure of the horizontal velocity history of the neutral particle during $0<t<5$; (c) Variations of drag force of the neutral particle; (d) Smoothed drag force curve. In (c, d), the negative values indicate resistance and the positive values indicate accelerating force.

Fig. 6 depicts the collision process between a hydrophobic particle and a droplet. The contact angle of the droplet on the hydrophobic particle reads 60 degree. Compared to their neutral counterpart (see Fig. 4), during $0<t<2.5$ the droplet stretches more significantly. When $t>2.5$ the droplet and the hydrophobic particle bear a repulsion force and thus they separate from each other slowly. During the separating process, the area of the droplet attached to the hydrophobic particle begins to decrease, and eventually the droplet departs away from the hydrophobic particle with 
a sharp tail. After the detachment, the droplet recovers its origin circular shape and leaves away from the hydrophobic particle.

Fig. 7 illustrates the collision process between a hydrophilic particle and a droplet. Compared to their neutral and hydrophobic counterparts at $t=2.5$ (see Fig. 4 and Fig. 6 ), the left side of the droplet is completely attached to the hydrophilic particle surface. Then the hydrophilic particle begins to penetrate the droplet, thus the droplet is split into two fragments, both of which are attached to the hydrophilic particle surface. The two fragments slip on the surface of the hydrophilic particle, and finally two small droplets are formed and keep attaching to the particle surface.

Fig. 8 (a) show the comparison of particle's horizontal velocity among these three cases. For the neutral particle, its horizontal velocity decreases to 0.04 at $t=2.0$. After that it fluctuates weakly and is in the range of 0.04-0.1. For the hydrophobic particle, its horizontal velocity decreases to 0.0 at $t=2.5$, and continues to decrease until $t=5.2$, which leads to a negative value. Later it begins to increase up to -0.43 at $t=12.5$, which implies that the solid particle completely exchange its momentum with the droplet and surrounding fluid. For the hydrophilic particle, its horizontal velocity experiences four stages in which its velocity increases and decreases alternatively and obviously. Finally its velocity approaches to 0.08 . Fig. 8 (b) depicts the variations of smoothed drag forces exerting on the particles with different particle wettability. Compared to its neutral and hydrophobic counterparts, the smoothed drag force exerting on the hydrophilic particle experiences more significant fluctuations. During $2<t<4$ the hydrophilic particle experiences a strong accelerating force and it is sucked into the droplet due to the hydrophilic solid surface (see Fig. 7). Thus its horizontal velocity increases sharply. During $4<t<6$ the two fragments originated from the droplet are observed on the hydrophilic particle surface, which causes an additional hydrodynamic resistance exerting on the hydrophilic particle. When $t>6$ the two fragments form two small droplets and thus the hydrophilic particle experiences a relatively weak oscillating force. For the hydrophobic particle, during $2<t<4$ the interaction behaves like a resistance force, resulting in a velocity decreasing process (see Fig. 8(a)). In addition, according to Fig. 8 (b), one can observe that the difference of smoothed drag force between a neutral and a hydrophobic particle is very small.
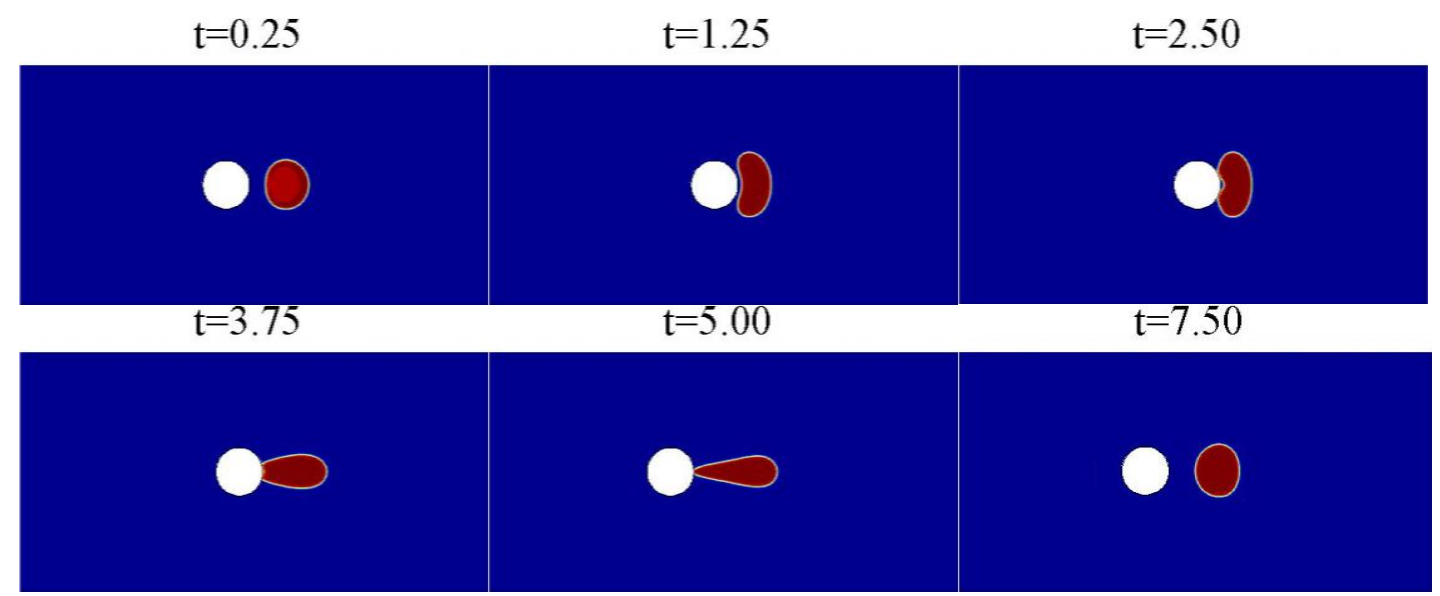

Fig. 6 The collision process between a hydrophobic particle $\left(\theta_{\mathrm{ca}}=60^{\circ}\right)$ and a liquid droplet at $B=0.0$, in which the white cylinder represents the particle and the red deformable represents the droplet. 


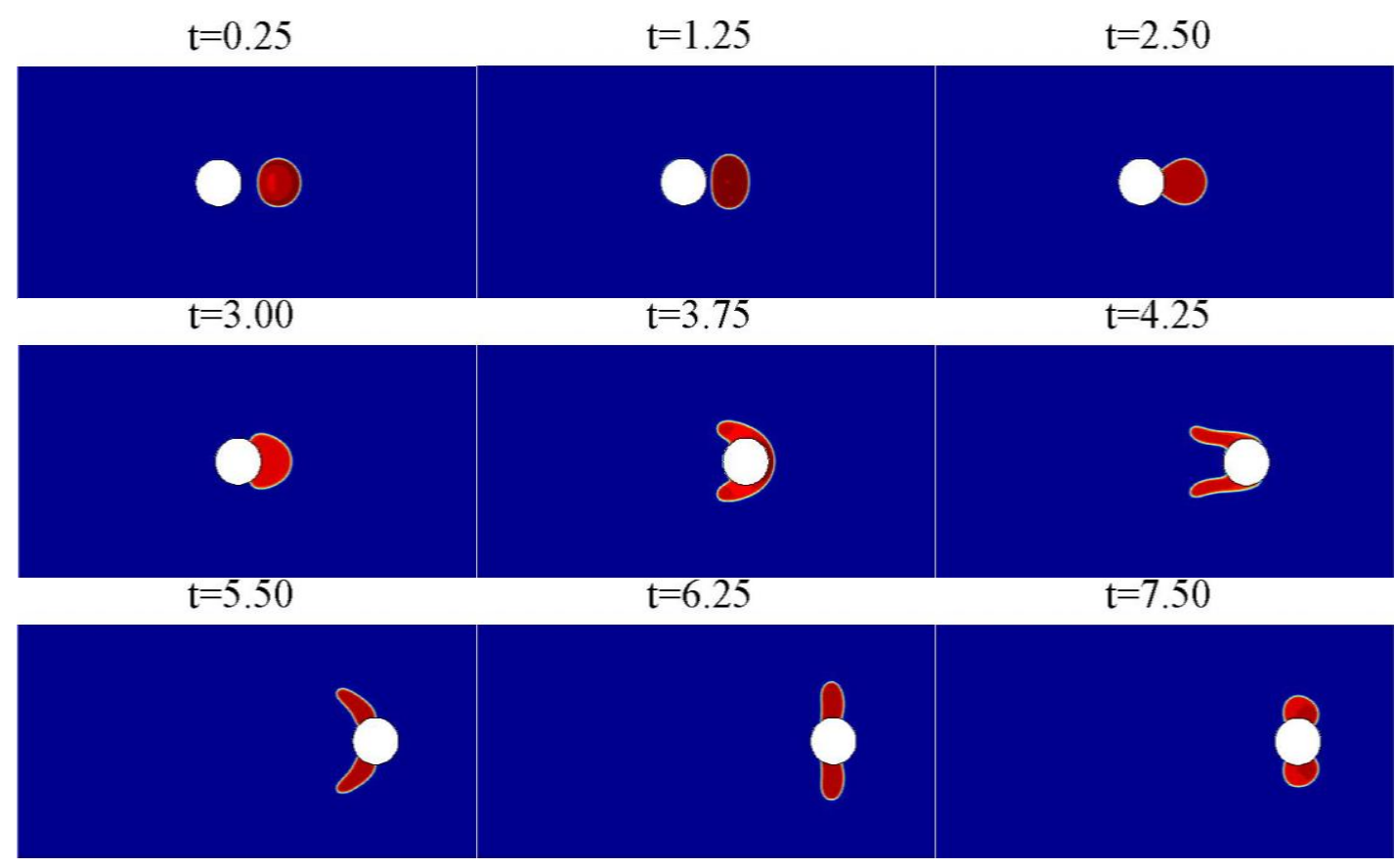

Fig. 7 The collision process between a hydrophilic particle $\left(\theta_{\mathrm{ca}}=120^{\circ}\right)$ and a liquid droplet at $B=0.0$, in which the white cylinder represents the particle and the red deformable represents the droplet.
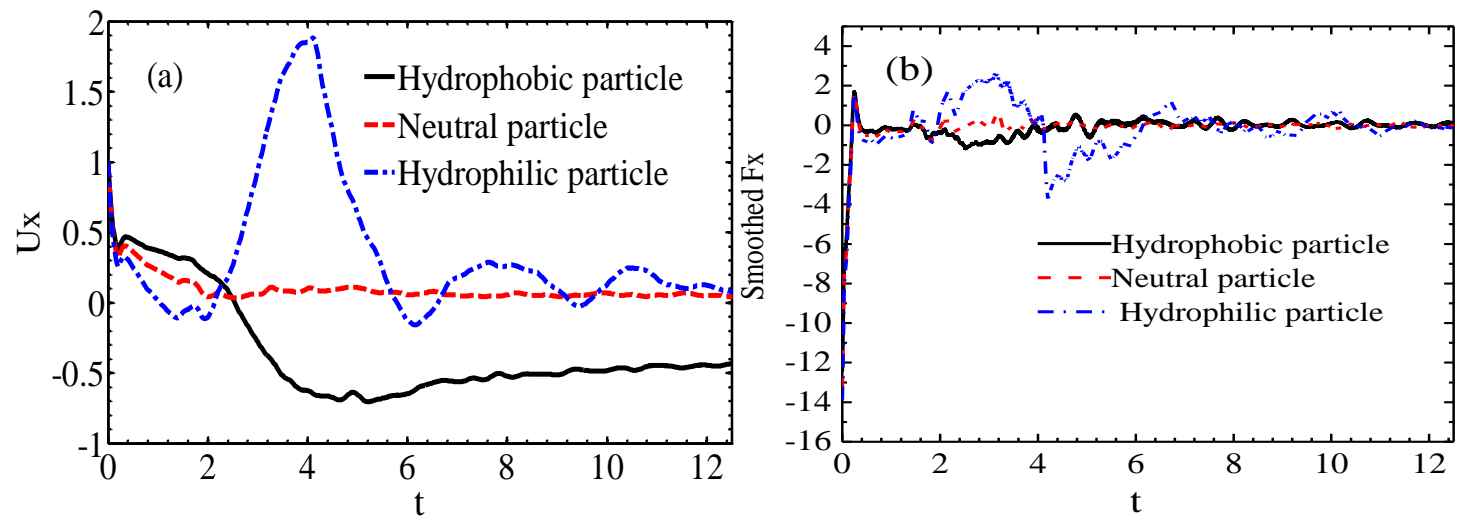

Fig. 8 Comparisons of (a) particle horizontal velocity and (b) smoothed drag forces exerting on particle among the neutral, hydrophilic and hydrophobic case at $B=0.0$.

When $B$ is not equal to 0 , eccentric collisions will happen. A small value of $B$ also can lead to agglomeration. Here the eccentric collision between a neutral particle and a droplet is taken as an example to analyze such interaction. Fig. 9 depicts the collision process between a neutral particle and a droplet when $B=0.2$. Compared to the case of $B=0$ discussed above, the droplet is attached to the upper right part of the neutral particle. When the neutral particle moves, the droplet slips on the particle's surface. In this paper, we use the droplet-to-particle angle $\left(\theta_{\mathrm{dp}}\right)$ to describe the relative position between the droplet and the particle. $\theta_{\mathrm{dp}}$ is the included angle between the horizontal line and the line connecting the center of particle and of droplet. $\theta_{\mathrm{dp}}$ is about $82^{\circ}$ at $t=37.5$. We compare the magnitude of $\theta_{\mathrm{dp}}$ at steady states for different $B$. It's found that the increasing of $B$ results in an increasing $\theta_{\mathrm{dp}}$. Fig. 10 shows the comparisons of particle's velocity for different $B$. As shown in Fig. 10 (a), the differences among the four cases are very little, although during $1<t<10$ the increasing of $B$ leads to the increasing of particle's horizontal velocity. However, as shown in 
Fig. 10 (b), the variations of particle's vertical velocity are more significant for the cases with larger $B$. In addition, it's worth noting that there are slight differences between the two cases where $B=0.2$ and 0.3 , and between the two cases of $B=0.5$ and 0.6. When $0<t<6.7$ the four vertical velocity curves look like sinusoids with oscillations. The result indicates that during the interaction process, the neutral particle firstly experiences repulsion force and then attraction force. Fig. 10 (c) and (d) illustrate the comparisons of smoothed horizontal and vertical force exerting on the neutral particle for these four cases. One can observe that there is no obvious differences among them. Here we take the case $B=0.2$ to discuss the variations of the force curves: during $0<t<5$ the horizontal and vertical forces both experience strong fluctuations, corresponding to the droplet attachment process. Then the two force curves oscillate weakly around 0 .

We also simulated the eccentric collisions (with a small $B$ ) of a hydrophobic and a hydrophilic particle. Their interaction processes are similar with their central collision counterparts $(B=0)$ discussed above. According to the above discussion, for the cases investigated in this study, it can be concluded that for a small $B$ (or the agglomeration regime), the wettability of a solid particle is the predominant parameter to determine interaction processes.
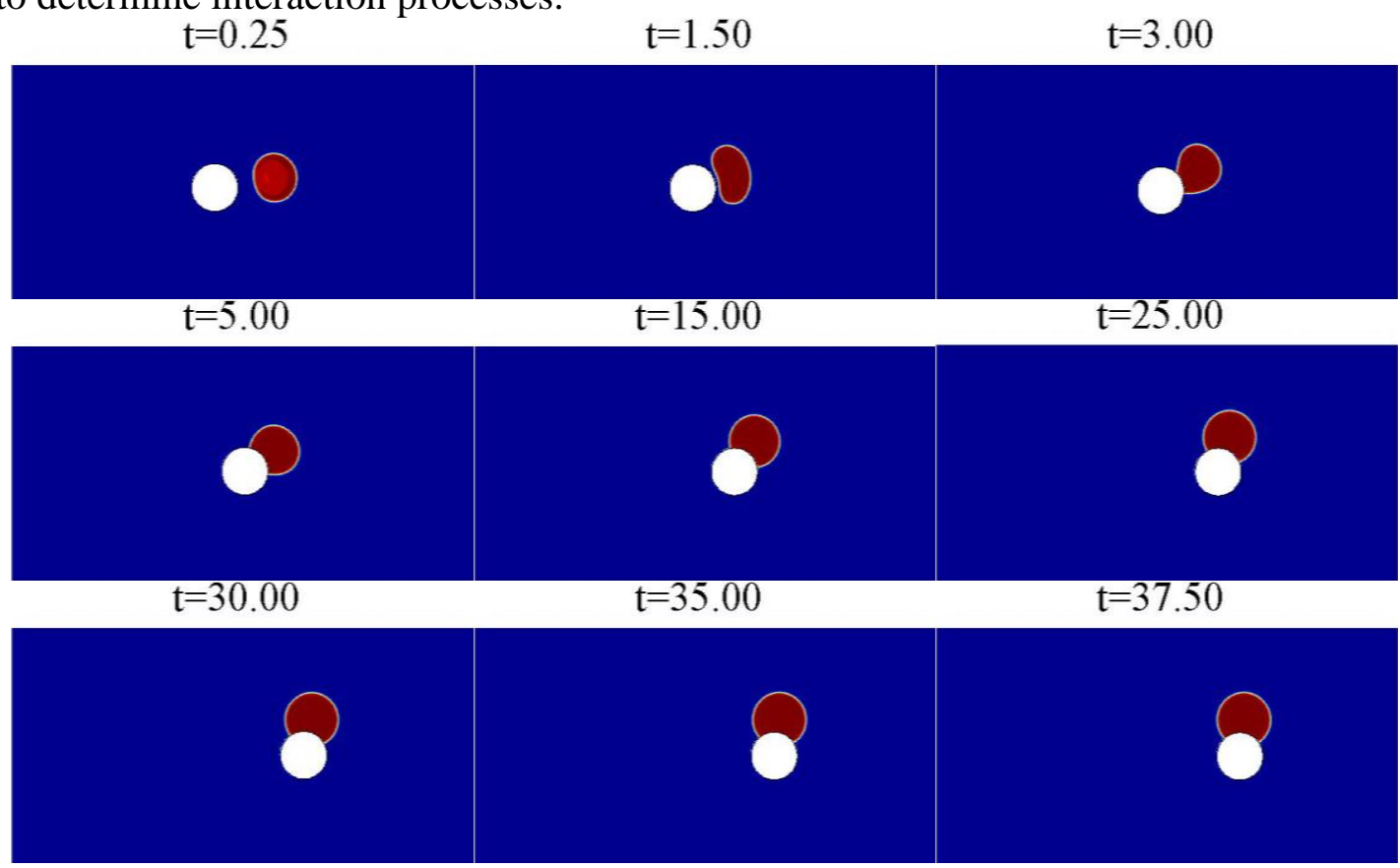

Fig. 9 The collision process between a neutral particle and a liquid droplet at $B=0.2$, in which the white cylinder represents the particle and the red deformable represents the droplet.
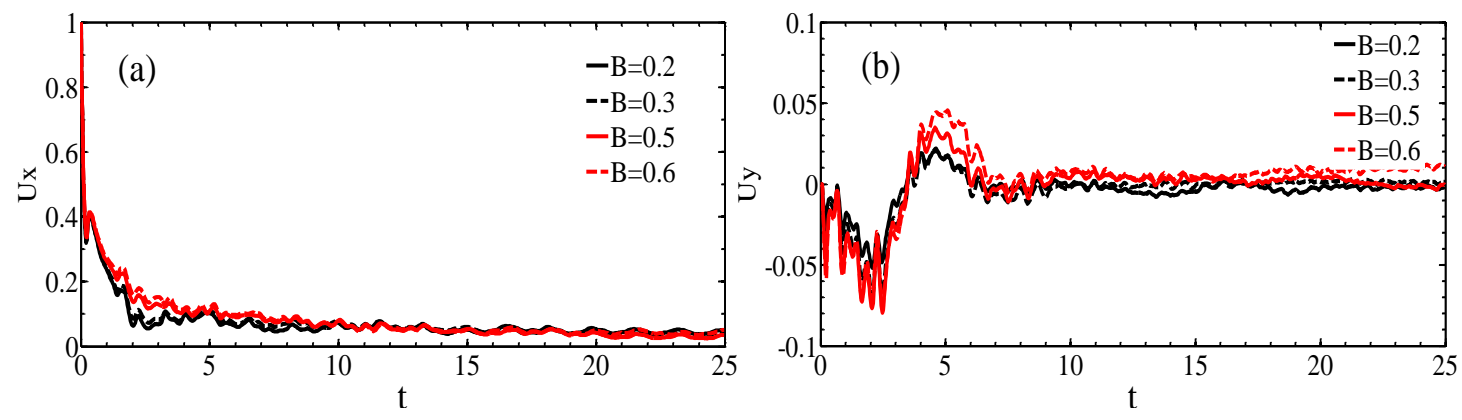

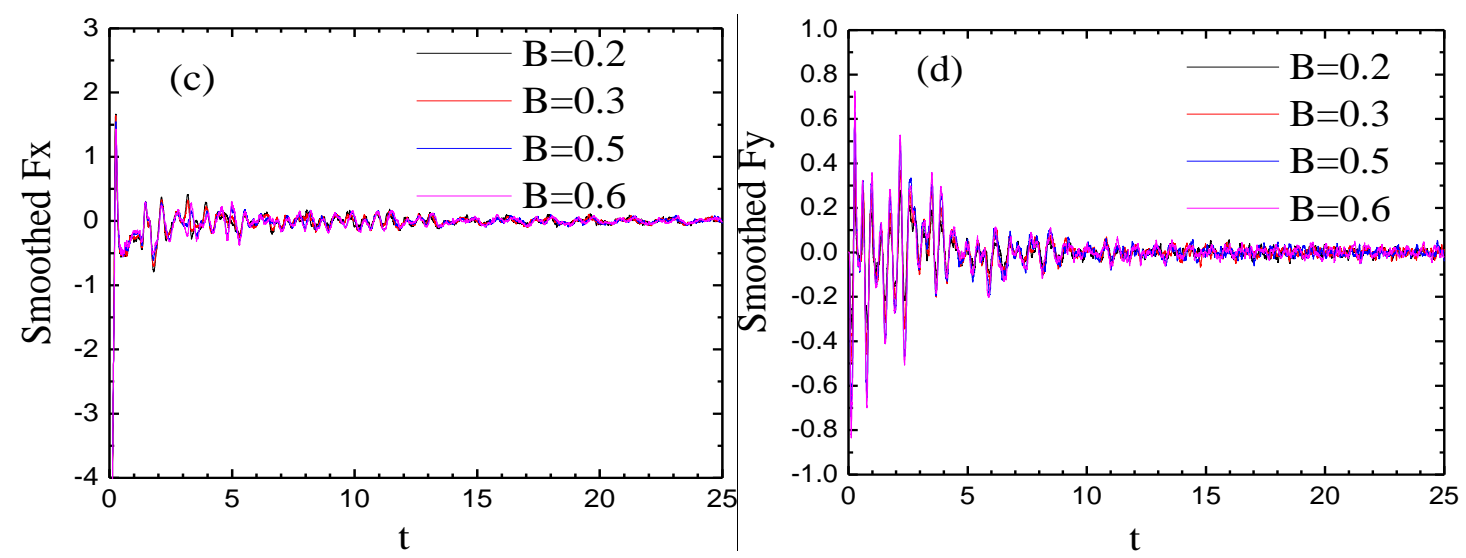

Fig. 10 Variations of (a) horizontal and (b) vertical velocities of the neutral particle at various $B$. Variations of smoothed (c) horizontal and (d) vertical force exerting on the neutral particle at various $B$.

\subsection{Separation regime}

When the eccentricity ratio $B$ is very large, a particle and a droplet will pass through the investigated domain without direct contact. In the present work such interaction process is classified by a separation regime. What should be stressed is that the present definition of separation regime is different from the stretching separation regime reported by Pawar et al. [34]. In the so-called stretching separation regime a droplet will directly contact a particle [34]. Fig. 11 depicts the interaction process between a neutral particle and a droplet at $B=0.7$. In the separation regime, the droplet stretches very little and passes over the particle without collision. Fig. 12 shows the variations of the particle's velocities for different $B$. We take the case $B=1.0$ as a representative to analyze it. As shown in Fig. 12 (a), the horizontal velocity experiences a sharp decreasing process until it decreases to 0.36. After a short-time increasing process it decreases again, but relatively slowly. As shown in Fig. 12 (b), the vertical velocity experiences strong oscillations during $0<t<5$. When $t>5$ the oscillations become weak and the vertical velocity approaches zero slowly. There are little differences among these three cases, especially for the particle's horizontal velocity. Compared to the agglomeration regime shown in Fig. 10 (b), during $0<t<5$ the particle's vertical velocity in the separation regime experiences more significant fluctuations. In addition, in the separation regime the particle's vertical velocity approaches to zero faster than its counterpart in the agglomeration regime.
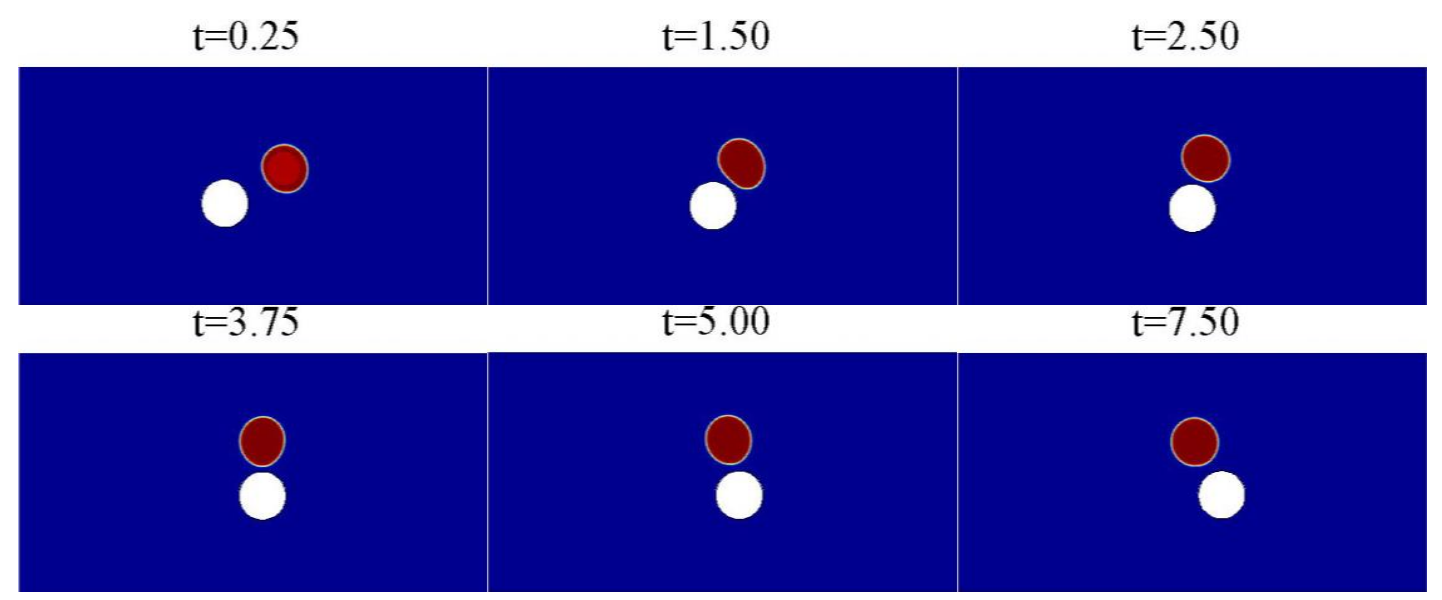


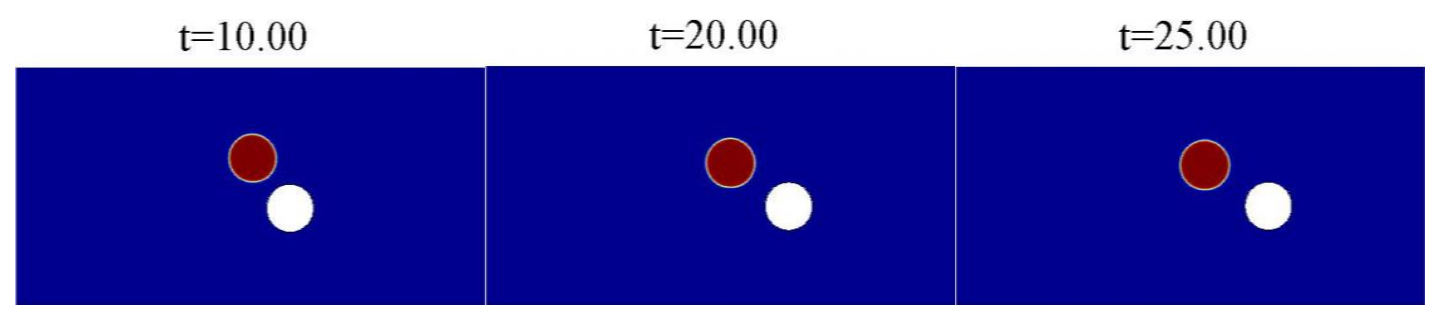

Fig. 11 The collision process between a neutral particle and a liquid droplet at $B=0.7$, in which the white cylinder represents the particle and the red deformable represents the droplet.
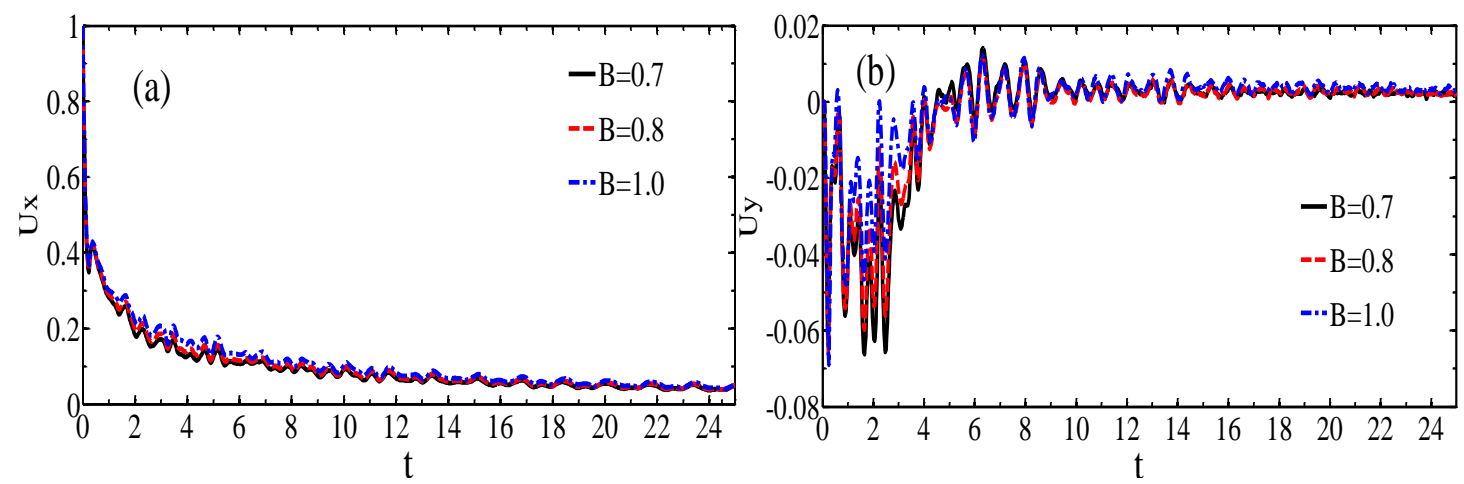

Fig. 12 Variations of (a) horizontal and (b) vertical velocities of neutral particle at various $B$.

Fig. 13 shows the interaction process between a hydrophobic particle and a liquid droplet at $B=0.7$. Compared to its neutral counterpart shown in Fig. 11, the droplet experiences a tumbling process and its obvious deformation occurs when it passes over the hydrophobic particle. Then the droplet runs away from the hydrophobic particle and recovers its origin circular shape. During the separation process, the distance between the droplet and the hydrophobic particle is much larger than its neutral counterpart. This phenomenon indicates that the droplet and the hydrophobic particle bear a repulsion force, which promotes their relative motion. In addition, obviously unlike its neutral counterpart, the hydrophobic particle moves in an oblique route.

Fig. 14 shows the interaction process between a hydrophilic particle and a liquid droplet at $B=0.7$. Compared to its neutral counterpart, the relative motion between the droplet and the hydrophilic particle is suppressed. Particularly when $t>20$, their relative motion almost disappears, and their relative position does not change any longer. This result indicates the droplet and the hydrophilic particle bear an attraction force, which hinders their relative motion.
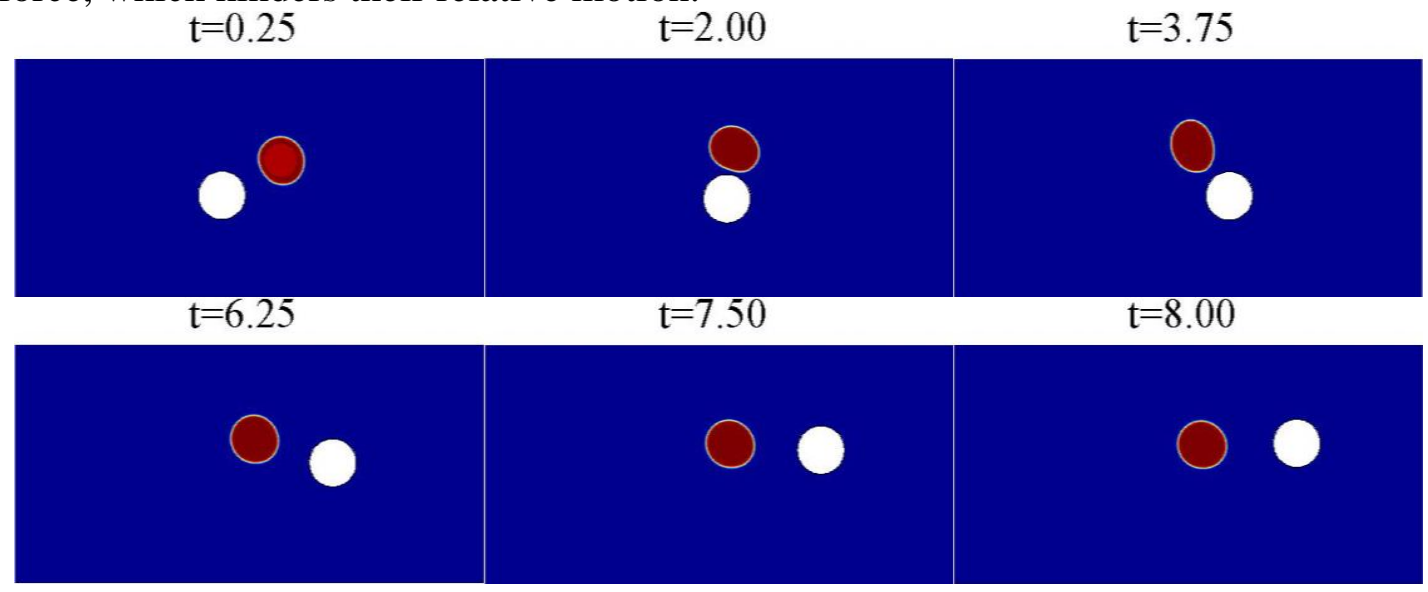


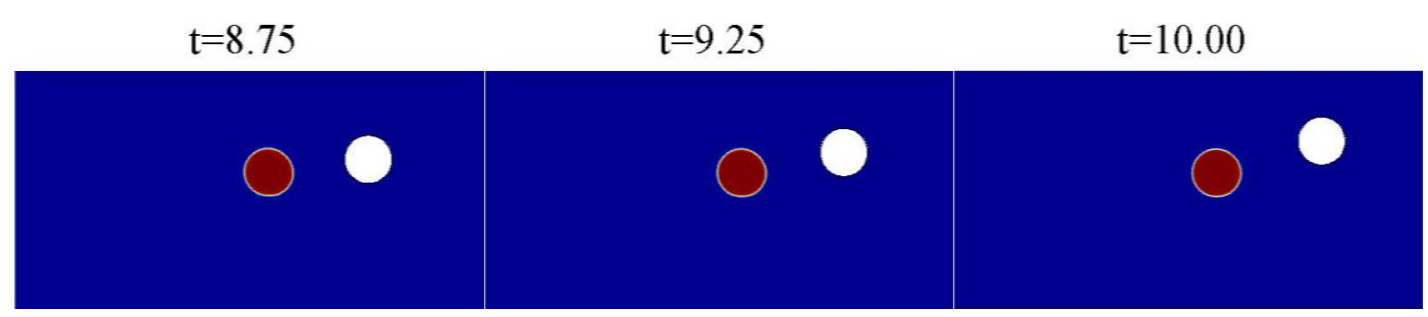

Fig. 13 The collision process between a hydrophobic particle $\left(\theta_{\mathrm{ca}}=60^{\circ}\right)$ and a liquid droplet at $B=0.7$, in which the white cylinder represents the particle and the red deformable represents the droplet.

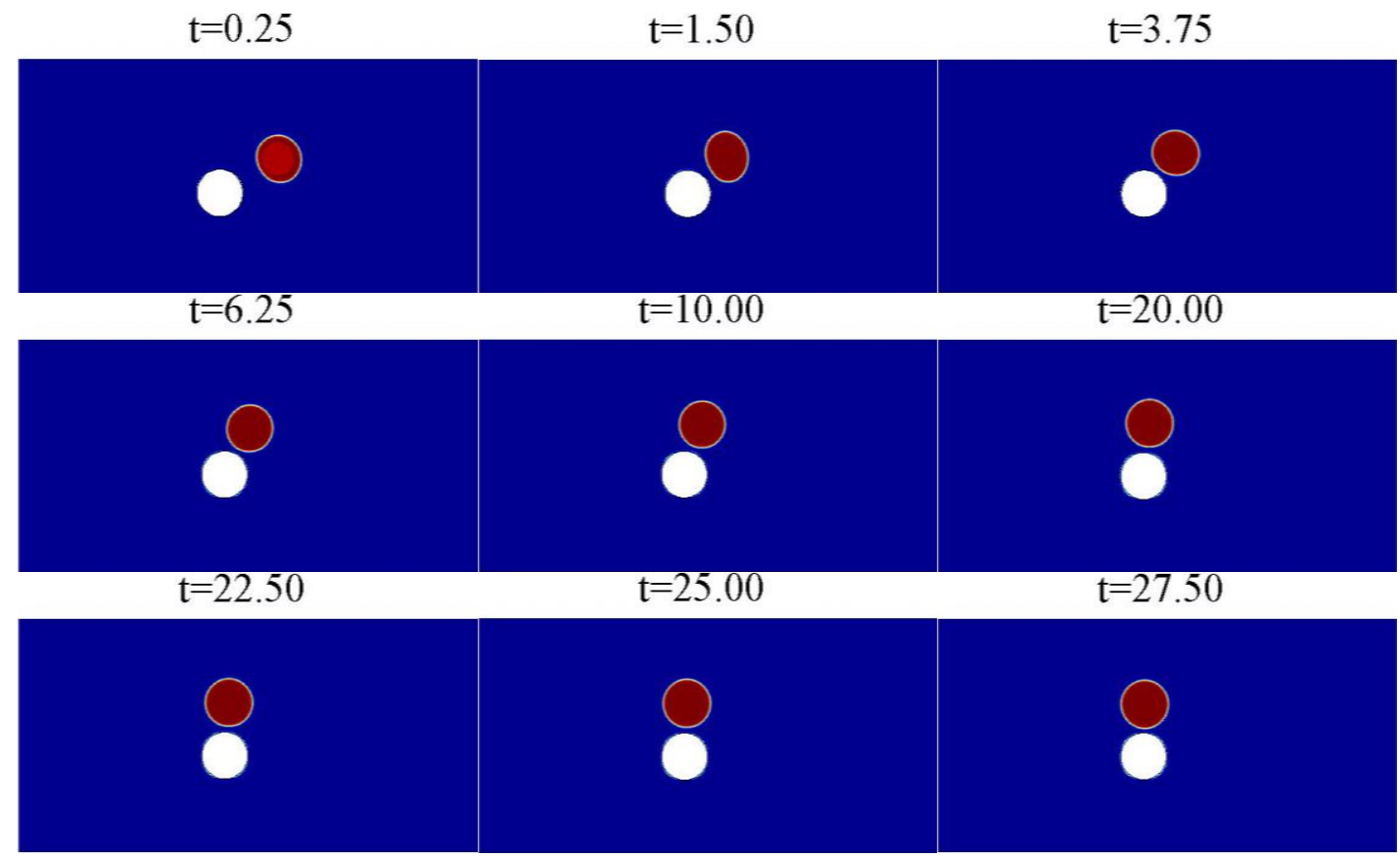

Fig. 14 The collision process between a hydrophilic particle $\left(\theta_{\mathrm{ca}}=120^{\circ}\right)$ and a liquid droplet at $B=0.7$, in which the white cylinder represents the particle and the red deformable represents the droplet.

\subsection{Effect of particle-to-droplet size ratio}

In this section, the effect of particle-to-droplet size ratio $\alpha=D_{\mathrm{p}} / D_{\mathrm{d}}$ on particle-droplet interaction is investigated by adjusting the size of the solid particle. In this section only a neutral solid particle is considered. The Weber number $W e=4 \rho_{\mathrm{d}} D_{\mathrm{d}} U_{0}^{2} / \sigma=33.3$ keeps constant but the capillary number $C a=2 \mu U_{0} D_{\mathrm{d}} / \sigma D_{\mathrm{p}}$ will change depending on $\alpha$. According to the above discussion on particle-droplet collisions at $\alpha=1.0$, we can deduce that a critical value of eccentricity ratio $\left(B_{c}\right)$ exists, beyond which the separation regime happens. Fig. 15 presents the distribution map of particle-droplet interaction regimes for various $\alpha(0<\alpha<2.5)$ and $B \quad(0<B \leqslant 1)$. Obviously, the agglomeration regime appears in the lower half of the map and the separation regime emerges in the upper half of the map. For each $\alpha$, there is a specific $B_{c}$. The peak value of $B_{c}$ is achieved for a middle $\alpha$ (for example $1.0 \leqslant \alpha \leqslant 1.6$ in this study) 


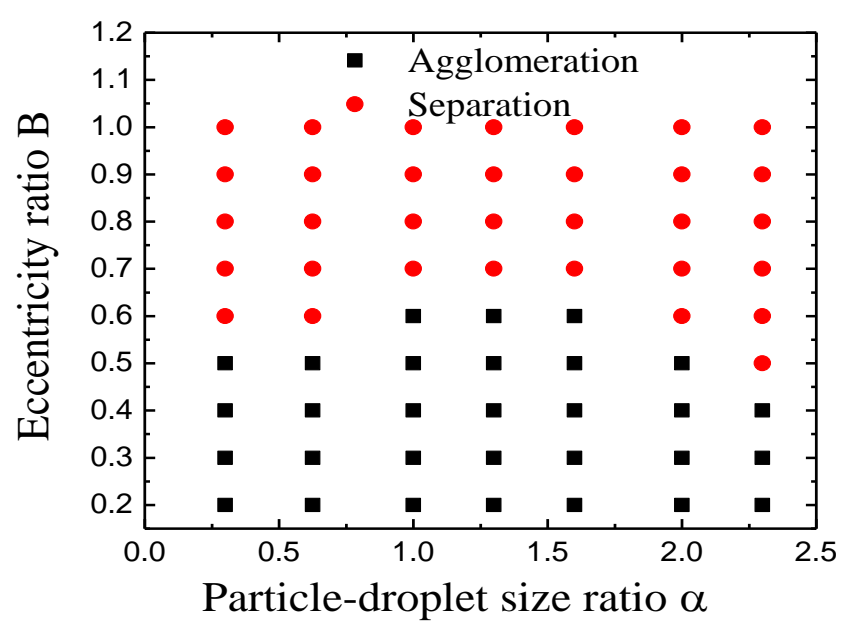

Fig. 15 A regime map for interaction between a freely moving particle and a freely moving droplet.

\section{Conclusion}

In this work, the interactions between a freely moving particle and a freely moving droplet are investigated. Depending on the magnitude of eccentricity ratio, the agglomeration and separation regime are observed, respectively. It is found that in the separation regime the droplet will pass over the particle without direct contact. In addition, the effect of particle-to-droplet size ratio on particle-droplet interaction are also studied. Some new phenomenon are observed. The conclusions of this work read:

(1) The effect of particle's wettability on the agglomeration regime is critical. For a neutral solid particle, the particle and the droplet can merge to form a stable combination with a contact angle of 90 on their interface. For a hydrophobic solid particle, the particle and the droplet bear a repulsion force after their agglomeration and thus they will separate from each other eventually. For a hydrophilic solid particle, the particle passes through the droplet, so the droplet is split into two fragments.

(2) The effect of particle's wettability on the separation regime is also critical. For a hydrophobic solid particle, the relative motion between the droplet and the particle is enhanced due to a repulsion force. For a hydrophilic solid particle, the relative motion between the droplet and the particle is suppressed due to an attraction force.

(3) The particle-to-droplet size ratio $\alpha$ influences the critical value of eccentricity ratio $B_{c}$ significantly. Generally, the peak value of $B_{c}$ is achieved for a middle $\alpha$.

\section{Acknowledgments}

This work has received funding from the Universidad Carlos III de Madrid, the European Unions Seventh Framework Programme for research, technological development and demonstration under grant agreement No. 600371, el Ministerio de Economa y Competitividad (COFUND2014-51509), el Ministerio de Educacin, cultura y Deporte (CEI-15-17) and Banco Santander. S. Chen would also acknowledge the support from the British Newton Alumni Fellowship Scheme.

\section{References}

[1] E. Tekin, P. J. Smith, U. S. Schubert, Inkjet printing as a deposition and patterning tool for polymers and inorganic particles, Soft Matter, 4 (4) (2008) 703-713.

[2] E. Dickinson, Food emulsions and foams: Stabilization by particles, Current Opinion in 
Colloid \& Interface Science, 15 (1-2) (2010) 40-49.

[3] T. N. Hunter, R. J. Pugh, G. V. Franks, G. J. Jameson, The role of particles in stabilising foams and emulsions, Advances in Colloid and Interface Science, 137 (2) (2008) 57-81.

[4] David E. Tambe, Mukul M. Sharma, Factors Controlling the Stability of Colloid-Stabilized Emulsions: I. An Experimental Investigation, Journal of Colloid and Interface Science, 157 (1) (1993) 244-253.

[5] F. Jansen, J. Harting, From Bijels to Pickering emulsions: a lattice Boltzmann study, Phys. Rev. E 83 (2011) 046707.

[6] C. K. Aidun, Y. N. Lu, E. J. Ding, Direct analysis of particulate suspensions with inertia using the discrete Boltzmann equation, Journal of Fluid Mechanics, 373 (1998) 287-311.

[7] A. J. C. Ladd, Numerical simulation of particulate suspensions via a discretized Boltmann equation, J. Fluid Mech., 309 (1994) 271-285.

[8] Z. G. Feng, E. E. Michaelides, The immersed boundary-lattice Boltzmann method for solving fluid-particles interaction problems, Journal of Computational Physics, 195 (2) (2004) 602-628.

[9] B. Yang, S. Chen, K. Liu, Direct numerical simulations of particle sedimentation with heat transfer using the Lattice Boltzmann method, International Journal of Heat and Mass Transfer, 104 (2017) 419-437.

[10] K. Stratford, I. Pagonabarraga, Parallel simulation of particle suspensions with the lattice Boltzmann method, Comput. Math. Appl., 55 (2008) 1585-1593.

[11] M. Orme, Experiments on droplet collisions, bounce, coalescence and disruption, Prog. Energy Combust. Sci. 23 (1997) 65-79.

[12] P.J. O'Rourke, F.V. Bracco, Modelling of drop interactions in thick sprays and a comparison with experiments, Proc. Inst. Mech. Eng. 9 (1980) 101-106.

[13] N. Ashgriz, J.Y. Poo, Coalescence and separation in binary collisions of liquid drops, J. Fluid Mech. 221 (1990) 183-204.

[14] S.L. Post, J. Abraham, Modeling the outcome of drop-drop collisions in Dieselsprays, Int. J. Multiphase Flow, 28 (2002) 997-1019.

[15] G.H. Ko, H.S. Ryou, Modeling of droplet collision-induced breakup process, Int. J. Multiphase Flow, 31 (2005) 723-738.

[16] T. Inamuro, R. Tomita, F. Ogino, Lattice Boltzmann simulation of drop deformation and breakup in shear flows, Int. J. Mod. Phys. B, 17 (2003) 21-26.

[17] A. Munnannur, R.D. Reitz, A new predictive model for the fragmenting and non-fragmenting binary droplet collisions, Int. J. Multiphase Flow, 33 (2007) 873-896.

[18] Z. Shen, Phase Transfer in a Collision Between a Droplet and Solid Spheres (MSc. Thesis) New Jersey Institute of Technology, New Jersey, 2008.

[19] Subhasish Mitra, Elham Doroodchi, Vishnu Pareek, Jyeshtharaj B. Joshi, Geoffrey M. Evans, Collision behaviour of a smaller particle into a larger stationary droplet, Advanced Powder Technology, 26 (1) (2015) 280-295.

[20] J. M. Gac, L. Gradon, Analytical investigation and numerical modeling of collisions between a droplet and a fiber, J Colloid Interface Sci, 369 (1) (2012) 419-425.

[21] J. M. Gac, L. Gradon, Lattice-Boltzmann modeling of collisions between droplets and particles, Colloids and Surfaces A: Physicochemical and Engineering Aspects, 441 (2014) 831-836.

[22] Y. Hardalupas, A.M.K. Taylor, J. Wilkins, Experimental investigation of sub-millimetre droplet impingement on to spherical surfaces, Int. J. Heat Fluid Flow, 20 (5) (1999) 477-485.

[23] S. Bakshi, I.V. Roisman, C. Tropea, Investigations on the impact of a drop onto a small spherical target, Phys. Fluids, 19 (3) (2007) 032102.

[24] Abbas Fakhari, Diogo Bolster, Diffuse interface modeling of three-phase contact line dynamics on curved boundaries: A lattice Boltzmann model for large density and viscosity ratios, Journal of Computational Physics, 334 (2017) 620-638.

[25] Ilias Malgarinos, Nikolaos Nikolopoulos, Manolis Gavaises, A numerical study on droplet-particle collision dynamics, International Journal of Heat and Fluid Flow, 61 (2016) 499-509. 
[26] Ilias Malgarinos, Nikolaos Nikolopoulos, Manolis Gavaises, Numerical investigation of heavy fuel droplet-particle collisions in the injection zone of a Fluid Catalytic Cracking reactor, Part I: Numerical model and 2D simulations, Fuel Processing Technology, 156 (2017) 317-330.

[27] Ilias Malgarinos, Nikolaos Nikolopoulos, Manolis Gavaises, Numerical investigation of heavy fuel droplet-particle collisions in the injection zone of a Fluid Catalytic Cracking reactor, part II: 3D simulations, Fuel Processing Technology, 156 (2017) 43-53.

[28] V.V. Dubrovsky, A.M. Podvysotsky, A.A. Shraiber, Particle interaction in three-phase

[29] polydisperse flows, Int. J. Multiphase Flow, 18 (3) (1992) 337-352.

[30] Niels G. Deen, Martin van Sint Annaland, J.A.M. Kuipers, Direct numerical simulation of complex multi-fluid flows using a combined front tracking and immersed boundary method, Chemical Engineering Science, 64 (2009) 2186-2201.

[31] Srdjan Sasic, Ebrahim Karimi Sibaki, Henrik Ström, Direct numerical simulation of a hydrodynamic interaction between settling particles and rising microbubbles, European Journal of Mechanics B/Fluids, 43 (2014) 65-75.

[32] Hiroyuki Kan, Hideya Nakamura, Satoru Watano, Effect of particle wettability on particle-particle adhesion of colliding particles through droplet, Powder Technology, 302 (2016) 406-413.

[33] Hiroyuki Kan, Hideya Nakamura, Satoru Watano, Effect of droplet size on particle-particle adhesion of colliding particles through droplet, Powder Technology, 321 (2017) 318-325.

[34] S. K. Pawar, F. Henrikson, G. Finotello, J. T. Padding, N. G. Deen, A. Jongsma, F. Innings, Jamh Kuipers, An experimental study of droplet-particle collisions, Powder Technology, 300 (2016) 157-163.

[35] Xiaowen Shan, Hudong Chen, Lattice Boltzmann model for simulating flows with multiple phases and components, Physical Review E, 47 (3) (1993) 1815-1819.

[36] X. Shan, Analysis and reduction of the spurious current in a class of multiphase lattice Boltzmann models, Phys Rev E Stat Nonlin Soft Matter Phys, 73 (2006) 047701.

[37] Nicos S. Martys, Hudong Chen, Simulation of multicomponent fluids in complex three-dimensional geometries by the lattice Boltzmann method, Physical Review E, 53 (1) (1996) 743-750.

[38] Z. Yu, H. Yang, L. S. Fan, Numerical simulation of bubble interactions using an adaptive lattice Boltzmann method, Chemical Engineering Science, 66 (14) (2011) 3441-3451.

[39] M.R. Swift, E. Orlandini, W.R. Osborn, J.M. Yeomans, Lattice Boltzmann simulations of liquid-gas and binary fluid systems, Phys. Rev. E, 54 (5) (1996) 5041.

[40] Porter M. L., E. T. Coon, Q. Kang, J. D. Moulton, J. W. Carey, Multicomponent interparticle-potential lattice Boltzmann model for fluids with large viscosity ratios, Phys Rev E, 86 (2012) 036701.

[41] A. S. Joshi, Y. Sun, Multiphase lattice Boltzmann method for particle suspensions, Phys Rev E Stat Nonlin Soft Matter Phys, 79 (2009) 066703.

[42] J.Y. Shao, C. Shu, Y.T. Chew, Development of an immersed boundary-phase field-lattice Boltzmann method for Neumann boundary condition to study contact line dynamics, J. Comput. Phys., 234 (2013) 8-32.

[43] Zhe Li, Julien Favier, Umberto D'Ortona, Sébastien Poncet, An immersed Lattice Boltzmann simulation of capillary interactions multi-component fluid flows, Journal of Computational Physics, 304 (2016) 424-440.

[44] J. Onishi, A. Kawasaki, Y. Chen, H. Ohashi, Lattice Boltzmann simulation of capillary interactions among colloidal particles, Comput. Math. Appl., 55 (2008) 1541-1553.

[45] Pawel Pieranski, Two-dimensional interfacial colloidal crystals, Physical Review Letters, 45 (7) (1980) 569-572. 